\section{The equivalence of target and nontarget processing in visual search*}

\author{
J. PATRICK CAVANAGH and WILLIAM G. CHASE $\dagger$ \\ Carnegie-Mellon University, Schenley Park, Pittsburgh, Pennsylvania 15213
}

A comparison of a forced-choice visual search task with an item recognition task did not support Neisser's (1967) hypothesis of a preattentive stage that processes targets and nontargets differentially. In the forced-choice condition, Ss indicated which of two items in a visual display was a target; in item recognition, Ss determined whether or not the single item in the visual display was a target. The size of the memorized set of possible targets was varied from one to six items for both tasks. Latencies increased linearly with memory set size in both conditions; the slopes for forced choice and item recognition were 41.8 and $27.9 \mathrm{msec}$ per item, respectively. The ratio of 1.38 between the two slopes was well fit by Sternberg's (1967) item recognition model, which predicts a ratio of 1.50 . This evidence supported the hypothesis that the identification or "standing out" of targets, as compared to "blurred" nontargets, in visual search occurs after both the encoding and memory search processes have terminated.

Visual search experiments (Neisser, 1964, 1967) have given evidence for a parallel memory search process, while item recognition studies (Sternberg, 1966, 1967) have supported a serial mechanism. Neisser has outlined a two-stage search theory that, in conjunction with the different demand characteristics of the two paradigms, accommodates these conflicting results.

The preliminary stage proposed by Neisser involves rapid, fairly inaccurate "preattentive" processing. Operating on easily discriminable physical features of the stimuli, the preliminary stage can, after sufficient practice, detect multiple targets in parallel. The subsequent "focal attentive" stage is a slower serial mechanism, acting upon less discriminable features of the stimuli.

Neisser argues that in visual search, where only targets require responses, the preliminary stage rejects nontargets but allows targets to pass through and be "identified." This hypothesis is based partly on Ss" reports that they hardly "see" the irrelevant letters-these are merely a "blur" from which the target letter "stands out" (Neisser, 1964). In item recognition, on the other hand, where both targets and nontargets require responses, both pass through the preliminary stage to be identified and matched serially against each memory item.

Sternberg (1967) has developed a quantitative model of the search process

\footnotetext{
* This research was supported by Public Health Research Grant MH-07722 from the National Institute of Mental Health.

$\dagger$ Requests for reprints should be sent to William G. Chase, Department of Psychology Carnegie-Mellon University, Pittsburgh, Pennsylvania 15213.
}

which postulates the same mechanism for both visual search and item recognition tasks. The basis of the model is a serial comparison process, with an exhaustive memory scan coupled with a self-terminating visual scan. ${ }^{1}$ Each item in the visual display is, in turn, exhaustively compared with the items in the memory set. When a match is found, the search terminates and no further visual items are processed. If there are d items in the visual display and the target location in the display is random, then an average of $(d+1) / 2$ of the visual items must be matched exhaustively against the s items in the memory set before a target is detected. If $b$ is the time required for a single comparison, then the average total comparison time for a positive response is $s \cdot[(d+1) / 2] \cdot b$. If, however, no match has been found after all the visual items have been processed, then a negative response is initiated. In this case, all visual items have been compared against all memory items, and the total comparison time is $s=d \cdot b$. In this model no mention is made of different demand characteristics for the two tasks.

The present experiment was designed to compare Neisser's and Sternberg's theories with two tasks requiring different demand characteristics: Sternberg's item recognition task and a forced-choice visual search task. In the item recognition task, Ss indicated whether or not a visually presented stimulus item was a target by pressing a positive or negative response key. The forced-choice task required Ss to indicate which of two visually displayed stimulus items was a target by pressing either a left - or a right-hand response key corresponding to the spatial location of the target. The latter task followed the demand characteristics of Neisser's visual search paradigm in that it required responses for targets only.

Applying Sternberg's model to these tasks produces the following predictions. In the item recognition task, with a single item in the visual display, $d$ is equal to 1 . Latencies, averaged over positive and negative stimuli, are then predicted from the following equation:

$$
\overline{\mathrm{RT}}_{\mathrm{ir}}=\mathrm{a}_{\mathrm{ir}}+\mathrm{b} \cdot \mathrm{s}
$$

The forced-choice task, under Sternberg's model, becomes functionally equivalent to an item recognition task with a positive stimulus set of two items $(d=2$, the search will terminate after processing the target item). Only the response process is different: Once the target is detected, a response corresponding to its spatial location, rather than a "YES" response, is initjated. Latencies are therefore predicted by the following expression:

$$
\overline{\mathrm{RT}}_{\mathrm{fc}}=a_{\mathrm{fc}}+1.5 \mathrm{~b} \cdot \mathrm{s}
$$

Neisser's theory as outlined here yields the sarne predictions as Sternberg's model in the item recognition task because the preattentive stage plays no differential role in the processing. With forced choice, however, where only targets elicit responses, the preliminary stage rejects nontargets, allowing potential targets to proceed to the serial search stage and identification. Thus, if the preliminary screening of nontargets is completely effective, target items will consistently "jump out" and only a single item will be matched against the memory set on each trial. The relationship of latencies to memory set size (s) in this case is, therefore, the same as for the item recognition task:

$$
\overline{\mathrm{RT}}_{\mathrm{fc}}=\mathrm{a}_{\mathrm{fc}}+\mathrm{b} \cdot \mathrm{s}
$$

The preattentive stage is, however, inherently error prone. The incorrect selection of the nontarget item for processing in the serial stage then requires the additional selection of the remaining item, increasing the average number of items that must be processed serially. If the preattentive stage is totally ineffective (i.e., it selects at random), then the average number of items processed serially will rise to the number predicted by Sternberg's model (1.5), and no differential processing of targets will be obtained. In this case, the identification phenomenon will not be at all consistent-both the target and the nontarget will be identified on fully half the trials.

If Neisser's hypothesis of differential processing of targets and nontargets holds, 


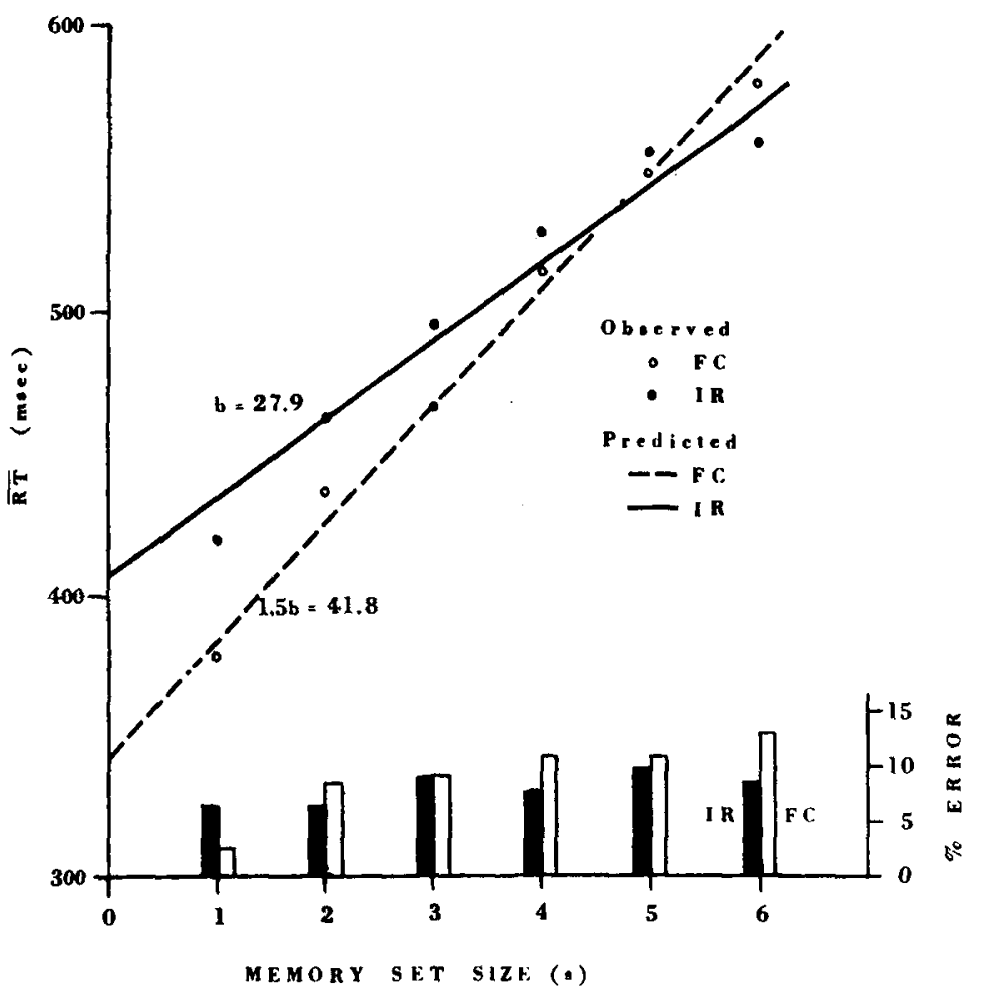

Fig. 1. Observed and predicted reaction times and percentage incorrect responses for item recognition (IR) and forced choice (FC) tasks.

a consistent target identification phenomenon should be reported, and the slope of the $\overline{\mathrm{RT}}$ vs memory set size relation should not be significantly greater (allowing for some inaccuracy in the preattentive stage) in the forced-choice than in the item recognition task.

A forced-choice slope of 1.5 times the item recognition slope will support Sternberg's model, in which targets and nontargets receive equivalent processing in both the encoding and memory search stages.

Finally, the different response compatibilities of the two tasks can be expected to significantly affect the reaction times. The forced-choice task involves a highly compatible spatial-spatial mapping of stimuli onto responses, whereas the item recognition task requires a relatively incompatible mapping (Fitts \& Posner, 1967, Chap. 6). The forced-choice intercept $\left(a_{f}\right)$ can thus be expected to be lower than the item recognition intercept $\left(a_{i r}\right)$.

\section{METHOD}

On each trial, $\mathrm{S}$ was shown from one to six letters of the alphabet to be memorized (memory set) and then a stimulus set to be searched. The task in the item recognition condition was to decide if the single item in the stimulus set was one of the letters in the memory set and to register the decision by pressing one of two response keys (positive or negative). In forced choice, the $\mathrm{S}$ was to indicate which of the two letters in the stimulus set was included in the memory set by pressing one of two response keys (left or right).

The memory and stimulus sets were drawn from the 26 letters of the alphabet. They were block capitals, subtending $18 \mathrm{~min}$ of visual angle when viewed on the tachistoscope display. The center-to-center distance of the stimulus items in the forced-choice task subtended $1 \mathrm{deg}$ of visual angle.

Two-hundred and sixteen memory sets were used. They varied in length from one to six different randomly selected letters, with 36 sets of each length. Target and nontarget stimulus items were equally probable in the item recognition task, while left and right target locations were equally probable in forced choice. The location of the target item in the memory set was varied equally over all serial positions, while the nontarget was chosen randomly from letters not in the memory set.

Sixteen Ss were run in both the item recognition and forced-choice conditions. The order of the two tasks was counterbalanced across Ss. The hands assigned to the positive and negative response keys in the item recognition task were counterbalanced across $\mathrm{Ss}$; in forced choice the left response key was pressed for a target on the left and the right key for a target on the right.

The Ss' initial instructions were to respond rapidly while making as few errors as possible. The 216 trials in each condition took roughly $1 \mathrm{~h}$ and were preceded by 12 practice trials. On each trial the memory set was displayed until $S$ pushed a ready key. After a 1-sec interval the stimulus set appeared and remained for $150 \mathrm{msec}$. The $S$ was told, after he pressed a response $\mathrm{key}$, his reaction time and whether or not he was correct.

\section{RESULTS}

Reaction time was a linear function of memory set size for both conditions $(\mathrm{p}<.001)$, with the observed slope being $29.3 \mathrm{msec}$ per memory item for item recognition and $40.4 \mathrm{msec}$ for forced choice. The ratio of 1.38 between the two slopes represented a significant difference in linear trends ${ }^{2}(\mathrm{p}<.01)$, while the deviation of the observed ratio from the 1.5 predicted by Sternberg's model was not significant.

Despite the lack of support for Neisser's hypothesis, Ss reported a consistent strong phenomenon of target identification in the forced-choice task.

The least-squares fit of Sternberg's theory (Eqs. 1 and 2) to both tasks is shown in Fig. 1. The predicted latencies did not deviate significantly from the observed latencies; the model, with three parameters (time per comparison, b, and two intercepts, $a_{i r}$ and $a_{f c}$ ), obtained an RMSD of $9.4 \mathrm{msec}$ with $9 \mathrm{df}$. The predicted slopes were 27.9 in the item recognition condition and 41.8 in the forced choice.

The results in the item recognition task were, in good agreement with previous studies: The difference in slopes for positive and negative stimuli was not significant, and positive responses were, on the average, $23.9 \mathrm{msec}$ faster than nontarget responses. Consistent with the exhaustive memory search in both tasks, there was no significant effect of the serial position of the target in the memory set.

In the forced-choice task, the slope for a target on the left of the visual display was not significantly different from that for a target on the right, while the intercepts for the two target locations were virtually identical.

The $64.2-\mathrm{msec}$ difference in intercepts between the forced choice and item recognition tasks was highly significant $(\mathrm{p}<.01)$. This difference can be attributed to the compatibility of the stimulus response mapping in the forced choice as compared to the item recognition task. 


\section{DISCUSSION}

Results from previous item recognition studies that incorporated conditions comparable to the two tasks in the current study (Nickerson, 1966; Sternberg, 1967; Briggs \& Blaha, 1969) have been mixed. The ratio of the least-squares slope for a positive stimulus set of two items (comparable to the forced choice task) to that for a single stimulus item was 1.05 in Nickerson's study, 1.90 in Sternberg's, and 1.66 in Briggs and Blaha's. (The Briggs and Blaha study also reported the target identification phenomenon for positive stimulus sets.)

It would not be valid, however, to draw conclusions from these studies to Neisser's visual search paradigm, since the "demand characteristics" are different. The point of the present study is that when these demand characteristics are met-as in the forced choice task-the data are consistent with Sternberg's model. The results of the current study thus do not support Neisser's hypothesis of a preattentive screening of nontargets in visual search. The consistent subjective report of target identification indicates that this phenomenon is not related to a preliminary stage, but must occur after all encoding and memory search processes have terminated.

Neisser had taken the identification phenomenon as evidence of a preliminary analysis that could differentiate targets from nontargets, rejecting the latter and allowing the former to continue to higher level processing. In Sternberg's model, however, both targets and nontargets receive identical analysis. Target identification, then, is subsequent to rather than an integral part of, the search process.

There is no concrete evidence, then, for the activity of a preliminary stage when the stimulus items have no grossly differentiable physical characteristics. The ability, after sufficient practice, to search for multiple targets as rapidly as for a single target (Neisser, 1964) may result from a more efficient organization of the serial search process, perhaps initiating multiple searches in parallel, as proposed by Sternberg and Scarborough (1969).

\section{REFERENCES}

ATKINSON, R. C., HOLMGREN, J. E., \& JUOLA, J. F. Processing time as influenced by the number of elements in the visual display. Perception \& Psychophysics, 1969, 6, 321-326.

BRIGGS, G. E., \& BLAHA, J. Memory retrieval and central comparison times in information processing. Journal of Experimental Psychology, 1969, 79, 395-402.

CHASE, W. G., \& POSNER, M. I. The effect of auditory and visual confusability on visual and memory search tasks. Paper presented to the Midwestern Psychological Association, Chicago, May 1965.

FITTS, P. M., \& POSNER, M. I. Human performance. Belmont, Calif: Brooks/Cole, 1967.

NEISSER, U. Visual search. Scientific American, 1964, 210, 94-102.

NEISSER, U. Cognitive psychology. New York: Appleton-Century Crofts, 1967.

NICKERSON, R. S. Response times with a memory-dependent decision task. Journal of Experimental Psychology, 1966, 72, 761.769.
STERNBERG, S. High speed scanning in human memory. Science, 1966, 153, 652-654.

STERNBERG, S. Scanning a persisting visual image versus a memorized list. Paper presented to the Eastern Psychological Association, Boston, April 1967.

STERNBERG, S., \& SCARBOROUGH, D. L. Parallel testing of stimuli in visual search. Paper presented to the International Symposium on Visual Information Processing \& Control of Motor Activity, Sofia, Bulgaria, July 1969.

\section{NOTES}

1. Studies by Chase and Posner (1965) and Atkinson et al (1969), investigating the condition of a single item in memory and a variable number of items in the visual display, have found that search is exhaustive through the visual display, However, in analyzing the studies that involved a variable number of items in both the memory set and the visual display (Sternberg, 1967; Nickerson, 1966), Atkinson et al did not find conclusive evidence in favor of either type of search when there was only a single item in memory. In applying Sternberg's model to the present experiment, where the memory set varied from one to six items, search through the visual display was assumed to be self-terminating in the one relevant condition where this was questionable (in the forced choice task, with a single item in the memory set).

2. An alternative model, which can be rejected from the data, is that Ss attend to only one item in the forced-choice task and process the other stimulus by inference. According to this model, if the attended item is a target, Ss press the corresponding key; if the item is not a target, Ss press the opposite key. The slopes are predicted to be equal in the item recognition and the forced choice conditions under this alternative.

(Accepted for publication November I, 1970.) 